\title{
Comparison of ultrasonography with computed tomography in the diagnosis of incisional hernias
}

\author{
D. den Hartog • A. H. M. Dur • A. G. A. Kamphuis • \\ W. E. Tuinebreijer $\cdot$ R. W. Kreis
}

Received: 22 February 2008 / Accepted: 18 July 2008 / Published online: 8 August 2008

(C) The Author(s) 2008. This article is published with open access at Springerlink.com

\begin{abstract}
Background The objective of this study is to determine the reliability and validity of ultrasonography (US) in diagnosing incisional hernias in comparison with computed tomography (CT). The CT scans were assessed by two radiologists in order to estimate the inter-observer variation and twice by one radiologist to estimate the intra-observer variation. Patients were evaluated after reconstruction for an abdominal aortic aneurysm or an aortoiliac occlusion.

Methods Patients with a midline incision after undergoing reconstruction of an abdominal aortic aneurysm or aortoiliac occlusion were examined by CT scanning and US. Two radiologists evaluated the $\mathrm{CT}$ scans independently. One radiologist examined the $\mathrm{CT}$ scans twice. Discrepancies between the CT observations were resolved in a common evaluation session between the two radiologists.

Results After a mean follow-up of 3.4 years, 40 patients were imaged after a reconstructed abdominal aortic aneurysm ( $80 \%$ of the patients) or aortoiliac occlusion. The prevalence of incisional hernias was $24 / 40=60.0 \%$ with CT scanning as the diagnostic modality and $17 / 40=42.5 \%$ with US. The measure of agreement between CT scanning and US expressed as a Kappa statistic was 0.66 (95\% confidence interval [CI] $0.45-0.88$ ). The sensitivity of US examination when using $\mathrm{CT}$ as a comparison was $70.8 \%$, the specificity was $100 \%$, the predictive value of a positive US
\end{abstract}

D. den Hartog $(\bowtie)$ A. H. M. Dur .

W. E. Tuinebreijer $\cdot$ R. W. Kreis

Department of Surgery, Red Cross Hospital,

Vondellaan 13, 1942 LE Beverwijk, The Netherlands

e-mail: d.denhartog@erasmusmc.nl

A. G. A. Kamphuis

Department of Radiology, Red Cross Hospital,

Vondellaan 13, 1942 LE Beverwijk, The Netherlands was $100 \%$, and the predictive value of a negative US was $69.6 \%$. The likelihood ratio of a positive US was infinite and that of a negative US was 0.29 . The inter- and intraobserver Kappa statistics were 0.74 (CI 0.54-0.95) and 0.80 (CI 0.62-0.99), respectively.

Conclusions US imaging has a moderate sensitivity and negative predictive value, and a very good specificity and positive predictive value. Consistency of diagnosis, as determined by calculating the inter- and intra-observer Kappa statistics, was good. The incidence of incisional hernias is high after aortic reconstructions.

Keywords Ventral hernia $\cdot$ X-ray computed tomography $\cdot$ Endosonography

\section{Introduction}

Incisional hernias, ventral hernias that manifest themselves through an operation scar, are a serious common complication of abdominal surgery. Incisional hernias occur in 11-23\% of laparotomies and can give rise to serious morbidity, such as strangulation and incarceration [1]. Often, the diagnosis can be made on clinical examination. However, small hernias and hernias in obese patients can be difficult to diagnose.

Diagnostic tools such as ultrasonography (US) and computed tomography (CT) are commonly used for imaging hernias. Most incisional hernias noted at cross-sectional imaging are incidental findings encountered during radiological examination for unrelated clinical problems. However, accurate demonstration of the size and location of the hernial orifice may be useful in assessing the success of hernia repair. The accuracy of these methods and their place in the clinical management of hernias have not yet been fully determined. 
In cases in which there is clinical uncertainty of the diagnosis of an incisional hernia, US or CT scanning can be used. The validity and inter-observer reliability of CT in the diagnosis of incisional, inguinal, and femoral hernias have been described in a preliminary study of 24 patients [2]. In this study, the gold standard was the situation found at operation. For two observers, the sensitivity was 0.83 and 0.83 , the specificity 0.83 and 0.67 , the positive predictive value 0.94 and 0.88 , and the negative predictive value 0.63 and 0.5 , respectively. The inter-observer Kappa statistic was 0.87. Although the ultrasonographic features of ventral hernias have been described, the reliability and validity of US in the diagnosis of ventral hernias have not been systematically studied [3-5]. A literature search did not reveal the existence of any systematic comparisons of CT scanning and US for use in the diagnosis of incisional hernias. However, an observational study compared ultrasound with CT scanning without describing reliability and validity [6].

The objective of this study is to determine the reliability and validity of US in the diagnosis of incisional hernias. CT scanning was used as a comparison in the determination of the validity. A gold standard was lacking because these patients were not operated after the diagnosis of an incisional hernia. The study population was composed of a group of patients who had previously undergone open reconstruction for abdominal aortic aneurysm or aortoiliac occlusive disease. Patients with an abdominal aortic aneurysm have a high incidence of ventral hernias. For instance, in a study comparing US and magnetic resonance imaging (MRI), the incidence of incisional hernias was $31.7 \%$ after reconstruction for abdominal aortic aneurysm after a mean duration of follow-up of 48.6 months [7]. In a systematic literature review, the pooled incidence of a postoperative incision hernia was $21 \%$ in abdominal aortic aneurysm patients and $9.8 \%$ in patients with aortoiliac occlusive disease [8].

\section{Materials and methods}

Forty patients (38 men, two women) who had undergone reconstruction for an abdominal aortic aneurysm or an aortoiliac occlusion between January 2002 and December 2006 at one single institution were selected for this study through the hospital administration system. The operation was required to have occurred at least one year prior to the study, because most incisional hernias develop in the first year after surgery $[9,10]$. Patients had undergone surgery through a midline incision by one of the two vascular surgeons. No other inclusion or exclusion criteria were used. These patients were examined by both CT scanning and US in November and December 2007. All US examinations were done by the same radiologist. This radiologist and another evaluated the CT scan independently. These radiologists were blinded to the outcome of the other diagnostic modality. One radiologist assessed the CT scans twice, with an interval of 4 weeks between assessments. In a common evaluation session, the two radiologists resolved the discrepancies between the three CT observations in order to develop a standard for comparison.

US examinations were performed using high-end ultrasound equipment (Aplio XG, model SSA-796A, Toshiba Medical Systems Corporation 1385, Shimoishigami, Otawara-Shi, Tochigi-Ken 324-8550, Japan, and ATL 5000, Philips, ATL factories, Bothell, USA) and linear transducer 5-12 MHz.

CT scanning was performed on a four-slice helical CT system (Asteion, Toshiba Medical Systems Corporation 1385, Shimoishigami, Otawara-Shi, Tochigi-Ken 3248550, Japan) with the following protocols: $120 \mathrm{kVp}$, $200 \mathrm{~mA}, 0.75$-s scan time, 3-mm slice thickness, 5.5 pitch.

The statistical methods assessed were the sensitivity, specificity, positive and negative predictive values, and positive and negative likelihood ratios. The Kappa coefficient was used to estimate the inter-observer variation between the two radiologists who examined the CT scans independently and the intra-observer variation between the two observations of one radiologist. The Kappa coefficient is an expression of the reproducibility of test results and can range from +1 to -1 . If the agreement is perfect, the Kappa statistic has the value +1 . Kappa gives the degree of agreement that has occurred over and above that which would have occurred by chance alone.

\section{Results}

After a mean follow-up of 3.4 years (standard deviation [SD] = 1.6), 40 patients were imaged after reconstruction for an abdominal aortic aneurysm ( $80 \%$ of the patients) or an aortoiliac occlusion (20\%). Ninety-five percent of the patients were male. The mean age was 72.5 years $(\mathrm{SD}=8.9)$. The prevalence of incisional hernias with $\mathrm{CT}$ scanning as the diagnostic modality after achieving consensus between the two radiologists was $24 / 40=60.0 \%$. The prevalence was $59.4 \%$ in the abdominal aortic aneurysm group and $62.5 \%$ in the occlusive disease group. With US as the diagnostic modality, the prevalence was 17/ $40=42.5 \%$.

In Table 1, the results of the CT scan and US are presented in cross-tabular form. CT scanning revealed the presence of seven hernias that were not found during US imaging, while on US, no hernia was seen that was not found on CT scanning. The measure of agreement between CT scanning and US, expressed as a Kappa statistic, was $0.66(95 \%$ confidence interval $[\mathrm{CI}] 0.45-0.88)$. 
Table 1 Results of computed tomography (CT) scanning and ultrasonography (US) in the diagnosis of incisional hernias

\begin{tabular}{lccc}
\hline & $\begin{array}{l}\text { CT positive } \\
\text { for hernia }\end{array}$ & $\begin{array}{l}\text { CT negative } \\
\text { for hernia }\end{array}$ & Total \\
\hline Ultrasound positive for hernia & 17 & 0 & 17 \\
Ultrasound negative for hernia & 7 & 16 & 23 \\
Total & 24 & 16 & 40 \\
\hline
\end{tabular}

Table 2 Results of US in the diagnosis of incisional hernia when CT scanning is used as a comparison

\begin{tabular}{lll}
\hline & US & CT \\
\hline Incidence (incisional hernia) & $17 / 40=42.5 \%$ & $24 / 40=60.0 \%$ \\
Sensitivity & $17 / 24=70.8 \%$ & \\
Specificity & $16 / 16=100.0 \%$ & \\
Positive predictive value & $17 / 17=100.0 \%$ & \\
Negative predictive value & $16 / 23=69.6 \%$ \\
Likelihood ratio positive & $\sim$ \\
$\quad$ (sens. $\div$ - $1-$ spec.) & \\
Likelihood ratio negative & 0.29 \\
$\quad(1-$ sens. $\div$ spec.) & & \\
\hline
\end{tabular}

In Table 2, the results of US in the diagnosis of incisional hernia are presented using CT scanning as a comparison. The sensitivity of a US examination was $70.8 \%$ and the specificity was $100.0 \%$. The predictive value of a positive US was $100.0 \%$ and that of a negative US was $69.6 \%$. In other words, $100 \%$ of the patients with a positive US had a positive CT scan and $69.6 \%$ of the patients with a negative US had a negative CT scan.

The likelihood ratio of a positive US was infinite, which means that the probability of a positive US being associated with a positive CT scan is an infinite number of times greater than the probability of a positive US associated with a negative CT scan. The likelihood ratio of a negative US is 0.29 , which means that the probability of a negative US coupled with a positive CT scan is 0.29 less than the probability of a negative US associated with a negative CT scan.

Table 3 presents the results of the two radiologists in the diagnosis of incisional hernias with CT scanning. The inter-observer variation for CT scanning between the two radiologists expressed as a Kappa statistic was 0.74 (95\% CI 0.54-0.95).

Table 4 shows the results of one radiologist in the diagnosis of incisional hernias with CT scanning on two occasions, with an interval between assessments of 4 weeks. The intra-observer variation for CT scanning between the two examinations, expressed as a Kappa statistic, was 0.80 (95\% CI 0.62-0.99).
Table 3 Results of the CT scan for radiologists A and B as a measure of inter-observer variation

\begin{tabular}{lccc}
\hline Radiologist A & \multicolumn{2}{l}{ Radiologist B } & \\
\cline { 2 - 4 } & $\begin{array}{l}\text { CT positive } \\
\text { for hernia }\end{array}$ & $\begin{array}{l}\text { CT negative } \\
\text { for hernia }\end{array}$ & Total \\
\hline CT positive for hernia & 21 & 1 & 22 \\
CT negative for hernia & 4 & 14 & 18 \\
Total & 25 & 15 & 40 \\
\hline
\end{tabular}

Table 4 Results of the CT scan evaluated twice by one radiologist as a measure of intra-observer variation

\begin{tabular}{llll}
\hline Second occasion & \multicolumn{2}{l}{ First occasion } & \\
\cline { 2 - 4 } & $\begin{array}{l}\text { CT positive } \\
\text { for hernia }\end{array}$ & $\begin{array}{l}\text { CT negative } \\
\text { for hernia }\end{array}$ & Total \\
\hline CT positive for hernia & 19 & 1 & 20 \\
CT negative for hernia & 3 & 17 & 20 \\
Total & 22 & 18 & 40 \\
\hline
\end{tabular}

\section{Discussion}

The sensitivity and negative predictive value of US in the diagnosis of incisional hernia were moderate in this study, because US yielded seven false-negative cases in the 24 patients who were CT positive for incisional hernia (29.2\%). Nevertheless, the specificity and positive predictive value were very high, because no false-positive cases were diagnosed by US examination. Højer et al. [2] found a lower specificity but a higher sensitivity in the diagnosis of hernias by CT examination. However, they examined a smaller combined group of groin and incisional hernias. Moreover, their gold standard was the situation at operation and they used CT as their imaging study.

In our group of patients, the majority of whom had an abdominal aortic aneurysm reconstruction, we found a very high incidence $(60.0 \%)$ of incisional hernias after a mean follow-up of 3.4 years. In comparison, the pooled analysis of Takagi et al. yielded an incidence of $21 \%$ in a total of 719 abdominal aortic aneurysm reconstruction patients [8]. However, most of the diagnoses included in the analysis were made clinically. Musella et al. [7] found an incidence of $31.7 \%$ for incisional hernias after a follow-up of 4 years in their patients, who were diagnosed by MRI and US. They concluded that US was unreliable in the early detection of anterior wall hernias and that US was more accurate in detecting normal rather than abnormal abdominal walls. This conclusion contradicts with our finding of high specificity and moderate sensitivity of US imaging.

Rodriguez et al. [11] found an incidence of $23 \%$ for abdominal wall hernias with CT scanning after open 
abdominal aortic aneurysm repair. Importantly, they concluded that clinical events and reinterventions related to these radiographic abnormalities are rare and that only $8 \%$ of the patients had clinical evidence of an incisional hernia.

Our inter-observer Kappa statistic of 0.74 is lower than the value of 0.87 found by Højer et al. [2], but the confidence intervals are wide in studies with such small samples. The intra-observer consistency (Kappa $=0.80$ ) is satisfactory in the light of the difficulty of reproducible, reliable clinical measurements [12].

A drawback of our study is that no measurements of the size of the hernias was made. Therefore, the influence of the hernia size on the sensitivity could not be determined.

In conclusion, abdominal wall US is an effective method for identifying incisional hernias, but it is only moderately accurate in detecting normal abdominal walls. The interand intra-observer reliability of CT examination for the diagnosis of incisional hernias is sufficient.

Open Access This article is distributed under the terms of the Creative Commons Attribution Noncommercial License which permits any noncommercial use, distribution, and reproduction in any medium, provided the original author(s) and source are credited.

\section{References}

1. Cassar K, Munro A (2002) Surgical treatment of incisional hernia. Br J Surg 89(5):534-545
2. Højer A-M, Rygaard H, Jess P (1997) CT in the diagnosis of abdominal wall hernias: a preliminary study. Eur Radiol 7:14161418

3. Ishida H, Konno K, Hamashima Y, Naganuma H, Komatsuda T, Sato M, Ishida J, Masamune O (1998) Anterior abdominal wall pathologies detected by high-frequency annular array. Eur J Ultrasound 7:167-174

4. Deitch EA, Engel JM (1980) Ultrasonic diagnosis of surgical diseases of the anterior abdominal wall. Surg Gynecol Obstet 151:484-486

5. Young J, Gilbert AI, Graham MF (2007) The use of ultrasound in the diagnosis of abdominal wall hernias. Hernia 11:347-351

6. Yeh HC, Lehr-Janus C, Cohen BA, Rabinowitz JG (1984) Ultrasonography and CT of abdominal and inguinal hernias. J Clin Ultrasound 12:479-486

7. Musella M, Milone F, Chello M, Angelini P, Jovino R (2001) Magnetic resonance imaging and abdominal wall hernias in aortic surgery. J Am Coll Surg 193:392-395

8. Takagi H, Sugimoto M, Kato T, Matsuno Y, Umemoto T (2007) Postoperative incision hernia in patients with abdominal aortic aneurysm and aortoiliac occlusive disease: a systematic review. Eur J Vasc Endovasc Surg 33:177-181

9. Pollock AV, Evans M (1989) Early prediction of late incisional hernias. Br J Surg 76:953-954

10. Burger JWA, Lange JF, Halm JA, Kleinrensink GJ, Jeekel H (2005) Incisional hernia: early complication of abdominal surgery. World J Surg 29:1608-1613

11. Rodriguez HE, Matsumura JS, Morasch MD, Greenberg RK, Pearce WH (2004) Abdominal wall hernias after open abdominal aortic aneurysm repair: prospective radiographic detection and clinical implications. Vasc Endovascular Surg 38:237-240

12. Sackett DL, Haynes RB, Guyatt GH, Tugwell P (1991) Clinical epidemiology: a basic science for clinical medicine, 2nd edn. Little, Brown \& Company, Boston, pp 25-31 